\title{
GAP STRUCTURE OF COHERENT ARONSZAJN TREES
}

\author{
Carlos Martinez-Ranero and Stevo Todorcevic
}

\begin{abstract}
Assuming the Proper Forcing Axiom, we show (identifying trees that are finite shifts of each other) that the class of coherent Aronszajn trees is the unique $\aleph_{2}$ saturated linear order of cardinality $\aleph_{2}$.
\end{abstract}

\section{Introduction}

An Aronszajn tree is an uncountable tree in which all levels and chains are countable. They show that the König infinity lemma does not extend to the realm of uncountable trees, although these objects were first discovered by Aronszajn and Kurepa in in the early 1930's (see [1], p.96) while analyzing the famous problem of Suslin ([5]). Their study, both in and outside the contexts of Suslin's problem has played an important role in the development of set theory. The class of Aronszajn trees has, however, its own intrinsic interest independent of the fact that many prominent problems of set theory have their reformulations inside this class. The purpose of this paper is to add to the structure theory of Aronszajn trees developed by the second author in [6]. Recall that in general a rough classification result for a given class $\mathcal{K}$ of structures usually depends on a quasi-ordering $\preceq$ on this class, i.e. a transitive and reflexive relation with the property that the inequality $A \preceq B$ means that in some sense $A$ is simpler structure than $B$. Since trees are natural generalization of ordinals there is already quite natural quasi-ordering between trees that fits well the class $\mathcal{A}$. We say that a tree $T$ is smaller than $S$ and write $T \preceq S$ iff there is an strictly increasing function $f: T \rightarrow S$. In this paper we are interested in the structure theory of $\langle\mathcal{A}, \preceq\rangle$ where $\mathcal{A}$ denote the class of all Aronszajn trees (from now on A-trees in short). Recall also that the strength of a rough classification result for a given class $\mathcal{K}$ of structures depends not only how fine the relation $\preceq$ we choose but also on how fine description of the corresponding structure $\langle\mathcal{K}, \preceq\rangle$ we give. One of the most prominent global conditions generally considered as giving satisfactory rough classification result is the requirement of being a well-quasi-ordered set. Recall that a class $\mathcal{K}$ is well-quasi-ordered (w.q.o., in short) if for every infinite sequence $\left(A_{n}\right)$ of elements of $\mathcal{K}$ there exist $n<m$ such that $A_{n} \preceq A_{m}$. The sense of strength of the w.q.o.-outcome in a rough classification result comes from the fact that whenever $(\mathcal{K}, \preceq)$ is well-quasi-ordered then the complete invariants of the equivalence relation

Received by the editors September 23, 2010.

2010 Mathematics Subject Classification. Primary Set Theory; Secondary Logic.

Key words and phrases. Aronszajn, coherent, linear order, saturated, universal.

The first author gratefully acknowledges support from Conacyt grant 159553 and the second author acknowledges support from NSERC and CNRS.. 
$\equiv^{1}$ on $\mathcal{K}$ are only slightly more complicated than the ordinals. In [6], solving a longstanding open problem of Laver[2], the second author showed that the class of A-trees is not well-quasi-ordered under $\preceq$ as there exist large families of pairwise incomparable A-trees as well as infinite strictly decreasing sequences of A-trees. This is in contrast with the corresponding result about the class of Aronszajn lines which is well quasi-ordered under $\preceq$ as shown by the first author in [3]. Thus the result of [6] shows that the structure $\langle\mathcal{A}, \preceq\rangle$ is far too complex to have a satisfactory classification result and that we must restrict ourselves to some subclass of this class. It turns out that the most natural class in this context would is the class $\mathcal{C}$ of Lipschitz trees. Recall that for given trees $S$ and $T$, a partial level-preserving map $g: T \rightarrow S$ is Lipschitz if

$$
\Delta(x, y) \leqslant \Delta(g(x), g(y)) \text { for all } x, y \in \operatorname{dom}(g),
$$

where for $x, y \in T$, we set

$$
\Delta(x, y)=\operatorname{otp}\{z \in T: z \leqslant x \text { and } z \leqslant y\} .
$$

A Lipschitz tree, is an A-tree tree $T$ with the property that every uncountable partial level-preserving map from $T$ into $T$ is Lipschitz on an uncountable subset of its domain. It turns out that in context of the Proper Forcing Axiom (which as we shall see is the right context for this kind of rough classification result) every Lipschiz trees is coherent and vice versa. We recall that an A-tree $T$ is said to be a coherent tree if $T$ can be represented as a downward closed subtree of the tree $\left(\omega^{<\omega_{1}}, \subseteq\right)$ in such a way that

$$
\{\xi \in \operatorname{dom}(t) \cap \operatorname{dom}(s): s(\xi) \neq t(\xi)\} \text { is finite for every } s, t \in T .
$$

The paper [6] of the second author shows that under the assumption of PFA the class $\mathcal{C}$ has some unexpected structure. For example, the class $\mathcal{C}$ is linearly ordered but not well-ordered under $\preceq$. Moreover, the class $\mathcal{C}$ is cofinal and coinitial in the class $\langle\mathcal{A}, \preceq\rangle$ of all A-trees. It is also proved in [6], using the same assumption PFA, that every Lipschitz tree is comparable with every other Aronszajn tree. Thus any rough classification result for the class $\mathcal{C}$ of Lipschitz trees is likely to give us some information about the whole class $\mathcal{A}$ of Aronszajn trees and vice versa any rough classification result about a sufficiently rich subclass of $\mathcal{A}$ must involve the class $\mathcal{C}$ in some way or the other. The paper [6] of the second author also proves that $\langle\mathcal{C}, \preceq\rangle$ is a discrete chain in the sense that every element $T$ of $\mathcal{C}$ has immediate successor $T^{(1)}$, the natural shit of $T$. Thus if we want to understand $\langle\mathcal{C}, \preceq\rangle$ it is natural to consider the quotient ordering $\langle\mathcal{C} / \mathbb{Z}, \preceq\rangle$ over the equivalence relation

$$
S \sim T(\bmod \mathbb{Z}) \text { iff }(\exists k \in \mathbb{Z}) S^{(k)}=T
$$

with countable and convex equivalence classes. If we are to provide some information about the linear order $\langle\mathcal{C} / \mathbb{Z}, \preceq\rangle$, the first thing is to understand its gap-structure. Indeed, we shall use $\mathrm{MA}_{\omega_{1}}$ to fill in any pre-gap whose sides have cardinalities at most $\aleph_{1}$. In other words, we show using $\mathrm{MA}_{\omega_{1}}$ that there are no $\left\langle\kappa, \lambda^{*}\right\rangle$-gaps in $\langle\mathcal{C} / \mathbb{Z}, \preceq\rangle$ whenever $\lambda, \kappa \in\left\{1, \omega, \omega_{1}\right\}$. From our results about gaps we shall infer that $\langle\mathcal{C}, \preceq\rangle$ is universal for all the linear orders of cardinality $\aleph_{2}$. Moreover, we shall prove, assuming PFA, that $\langle\mathcal{C} / \mathbb{Z}, \preceq\rangle$ is the unique saturated linear order of cardinality $\aleph_{2}$. We will make this more precise in the rest of the paper.

\footnotetext{
${ }^{1}$ Given by letting $A \equiv B$ iff $A \preceq B$ and $B \preceq A$.
} 


\section{Preliminaries}

In this section we will state mostly without proofs several results of the second author about the class of coherent Aronszajn trees, which will be needed in the rest of the paper. The reader is referred to Chapter 4 of [7] for their proofs.

Recall (see [6]), that a partial level-preserving map $g: T \rightarrow S$ is Lipschitz if

$$
\Delta(x, y) \leqslant \Delta(g(x), g(y))
$$

for all $x, y \in \operatorname{dom}(g)$, where $\Delta(x, y)=\operatorname{otp}\{z \in T: z \leqslant x$ and $z \leqslant y\}$.

Definition 1. A Lipschitz tree, is an uncountable tree $T$ with the property that every uncountable partial level-preserving map from $T$ into $T$ is Lipschitz in an uncountable subset of its domain.

As already indicated above, it was shown in [6] (see also Lemma 4.2.7 in [7]) that the class of Lipschitz trees agree with the class of coherent trees under the assumption $\mathrm{MA}_{\omega_{1}}$. The following lemma (see Lemmas 4.3.6 and 4.3.7 of [7]) shows one of the more prominent properties of Lipschitz trees and Lipschitz maps: in order to embed a given Lipschitz tree $S$ into another $T$, it suffices to find a Lipschitz map from an uncountable subset of $S$ into $T$.

Lemma 2. Assuming $\mathrm{MA}_{\omega_{1}}$, the following holds for every pair of coherent Aronszajn trees $S$ and $T$ :

(a) $S \preceq T$ if and only if there is an uncountable $X \subseteq S$ and a level-preserving map $f: X \rightarrow T$ such that $\Delta(x, y) \leqslant \Delta(f(x), f(y))$ (i.e. $f$ is a Lipschitz map).

(b) $S \prec T$ if and only if there is an uncountable subset $X \subseteq S$ and a levelpreserving map $f: X \rightarrow T$ so that $\Delta(x, y)<\Delta(f(x), f(y))$.

The following lemma (see Lemma 4.2.5 in [7]) is very useful when showing that certain partial orders satisfy the countable chain condition.

Lemma 3. Let $T$ be a coherent A-tree such that every uncountable subset of $T$ has an uncountable antichain. Let $n$ be a positive integer, and let $A$ be an uncountable family of pairwise-disjoint $n$-element subsets of $T$. Then there exits an uncountable $B \subset A$ such that $\Delta\left(a_{i}, b_{i}\right)=\Delta\left(a_{j}, b_{j}\right)$ for all $a \neq b$ in $B$ and $i, j<n .{ }^{2}$

The following result, appearing originally in [6] (see also Theorem 4.3 .10 in [7]), is one of the important applications of the last two lemmas.

Lemma 4. Assume $\mathrm{MA}_{\omega_{1}}$. Then for every pair $S$ and $T$ of coherent A-trees, either $S \preceq T$ or $T \preceq S$ holds.

From now on we assume that the trees $T$ are represented in such a way that every $t \in T_{\alpha}$ is simply a function from $\alpha$ into $\omega$. Let $\Lambda$ denote the set of all countable limit ordinals.

\footnotetext{
${ }^{2}$ Here, for a given $n$-element subset $a$ of $T$, we let $a=\left\{a_{1}, a_{2}, \ldots, a_{n-1}\right\}$ be its increasing enumeration according some fixed well-ordering of $T$.
} 
Definition 5. For an integer $m$ and a tree $T$, we let $T^{(m)}$ be its $m$-th shift, the downward closure of $\left\{t^{(m)}: t \in T\lceil\Lambda\}\right.$, where for a limit node $t$ of $T$, we let $t^{(m)}$ be the function with the same domain $\lambda$ as $t$ defined by,

$$
t^{(m)}(\xi)=t(\xi-m)
$$

when $\xi-m$ exists: otherwise, we let $t^{(m)}(\xi)=0$.

Note that a positive shift $T^{(m)}$ of any coherent tree $T$ is coherent (hence Lipschitz) and that the map $t \mapsto t^{(m)}$ is strictly increasing from $T$ into $T^{(m)}$. The case of negative shifts is more subtle as the negative shifts $T^{(-m)}$ are not always defined but they do behave as expected whenever defined ( see Lemma 4.3.21 [7]). The following lemma from [6] (see also Lemma 4.3.19 in [7]) is giving us the crucial property of the shift operation.

Lemma 6. Assuming $\mathrm{MA}_{\omega_{1}}$. For every pair $S$ and $T$ of Lipschitz trees, $S \prec T$ implies $S^{(1)} \preceq T$.

Note that this result together with Lemma 4 show that $\langle\mathcal{C} / \mathbb{Z}, \preceq\rangle$ is a well-defined quotient linear ordering with countable convex equivalence classes

We finish this section with a theorem that summarizes some results from [6] (see also Lemma 4.3.31, Lemma 4.3.33 and the proof of Theorem 4.3,35 [7]).

Theorem 7. Assume PFA. For any Aronszajn tree $T$ there exists two coherent Aronszajn trees $T_{0}$ and $T_{1}$ such that $T_{0}^{(m)} \prec T \prec T_{1}$ for all $n<\omega$. In particular, there are no maximal nor minimal Aronszajn trees and the quotient linear ordering $\langle\mathcal{C} / \mathbb{Z}, \preceq\rangle$ has no first element.

Remark 8. It is worth mentioning that the existence of $T_{1}$ follows from $\mathrm{MA}_{\omega_{1}}$, and it is not known if $\mathrm{MA}_{\omega_{1}}$ suffices for the existence of $T_{0}$.

\section{Posets for Interpolating Pre-Gaps}

Definition 9. If $A, B$ are subsets of $\omega_{1}$, then an $\left\langle A, B^{*}\right\rangle$-pre-gap on $\langle\mathcal{C}, \preceq\rangle$ is a pair of sequences of coherent Aronszajn trees

$$
\mathcal{T}_{A, B^{*}}=\left\langle T_{\xi}^{0}, T_{\eta}^{1}: \xi \in A, \eta \in B\right\rangle
$$

that satisfy the following requirements:

(1) $(\forall \xi \in A)(\forall \eta \in B) \quad T_{\xi}^{0} \prec T_{\eta}^{1}$,

(2) $\left(\forall \xi, \xi^{\prime} \in A\right) \xi<\xi^{\prime}$ implies $T_{\xi}^{0} \prec T_{\xi^{\prime}}^{0}$,

(3) $\left(\forall \eta, \eta^{\prime} \in B\right) \eta<\eta^{\prime}$ implies $T_{\eta^{\prime}}^{1} \prec T_{\eta}^{1}$.

We further assume that it satisfies the following technical condition:

(4) For all unequal pairs $(\xi, i) \neq(\eta, j)$ in $(A \cup B) \times 2$ we have that $T_{\xi}^{i} \cap T_{\eta}^{j}=\varnothing$.

We decide to use subsets of $\omega_{1}$ as opposed to cardinals in our definition of pre-gap to cover the case of strictly increasing sequence of trees as well as the case of strictly decreasing sequence of trees i.e. the cases $B=\varnothing$ and $A=\varnothing$, respectively. We shall associate to each $\left\langle A, B^{*}\right\rangle$-pre-gap $\mathcal{T}_{A, B^{*}}$ two forcing notions $\mathcal{P}\left(\mathcal{T}_{A, B^{*}}\right)$ and $\mathcal{P}^{*}\left(\mathcal{T}_{A, B^{*}}\right)$, which adjoin a coherent Aronszajn tree $T$ filling the pre-gap. The purpose of this section is to provide conditions on the pre-gap $\mathcal{T}_{A, B^{*}}$ for which the corresponding 
forcing notions satisfy the countable chain condition. In order to achieve this, we define some auxiliary notions associated to every pre-gap that will help us in the construction of the partial orders.

From our assumptions on the pre-gap, it follows that for every pre-gap $\mathcal{T}_{A, B^{*}}$ there is a well defined index function

given by

$$
\chi: \bigcup_{(\xi, i) \in(A \cup B) \times 2} T_{\xi}^{i} \rightarrow(A \cup B) \times 2
$$

$$
\chi(x)=(\xi, i) \text { if and only if } x \in T_{\xi}^{i} .
$$

Define a lexicographic ordering on $(A \cup B) \times 2$ by

$$
(\xi, i)<_{\text {lex }}(\eta, j) \text { iff } i<j \text { or } i=j=0 \text { and } \xi<\eta \text { or } i=j=1 \text { and } \xi>\eta \text {. }
$$

Using condition (4), for a given pre-gap $\mathcal{T}_{A, B^{*}}$, we can construct a subset $X=$ $X_{\mathcal{T}_{A, B^{*}}}$ of $\bigcup_{(\xi, i) \in(A \cup B) \times 2} T_{\xi}^{i}$ with the following properties:

(i) $X \cap T_{\xi}^{i}$ is uncountable for all $\xi \in A \cup B, i \in 2$,

(ii) $\left(\forall \alpha \in \omega_{1}\right)\left|X \cap \operatorname{Lev}_{\alpha}\left(T_{\xi}^{i}\right)\right| \leqslant 1$ for all $\xi \in A \cup B, i \in 2$,

(iii) $\left(\forall \alpha \in \omega_{1}\right) \quad\left|\left\{(\xi, i) \in(A \cup B) \times 2: X \cap \operatorname{Lev}_{\alpha}\left(T_{\xi}^{i}\right) \neq \varnothing\right\}\right| \leqslant 1$ and

(iv) $(\forall \xi \in A \cup B)(\forall i<2)\left(\forall x, y \in X \cap T_{\xi}^{i}\right) \Delta(x, y)>\omega$.

For each pre-gap $\mathcal{T}_{A, B^{*}}$, more precisely for each $X_{\mathcal{T}_{A, B^{*}}}$, defined as above, we construct the partial orders $\mathcal{P}\left(\mathcal{T}_{A, B^{*}}\right)$ and $\mathcal{P}\left(\mathcal{T}_{A, B^{*}}\right)$ as follows.

Definition 10. Let $\mathcal{T}_{A, B^{*}}$ be a given pre-gap and let $X=X_{\mathcal{T}_{A, B^{*}}}$. We define the partial order $\mathcal{P}=\mathcal{P}\left(\mathcal{T}_{A, B^{*}}\right)$ as follows: Let $\mathcal{P}$ be the poset of all partial finite mappings $p: X \times \omega_{1} \rightarrow \omega$ with the following properties:

(1) $\xi<h t(x)$ for all $(x, \xi) \in \operatorname{dom}(p)$,

(2) $\forall x, y \in \operatorname{dom}_{0}(p)^{3}$ and $\forall \xi<h t(x), h t(y)[(x, \xi) \in \operatorname{dom}(p)$ if and only if $(y, \xi) \in$ $\operatorname{dom}(p)]$,

(3) For all $x, y \in \operatorname{dom}_{0}(p)$,

(a) If $\chi(x)=\chi(y)=(\eta, 0)$ for some $\eta$, then

$$
[(x, \xi),(y, \xi) \in \operatorname{dom}(p), \xi<\Delta(x, y) \text { implies } p(x, \xi)=p(y, \xi)] .
$$

(b) If $\chi(x)=\chi(y)=(\eta, 1)$ for some $\eta$, then there exist

$$
\xi \leqslant \Delta(x, y) \text { such that } p(x, \xi) \neq p(y, \xi) \text {. }
$$

We let $p$ extend $q$ if $p$ extend $q$ as a function and

(4) $p(x, \xi)=p(y, \xi)$ for all $x, y \in \operatorname{dom}_{0}(q)$ and $\xi<\min \{h t(x), h t(y)\}, \xi \notin$ $\operatorname{dom}_{1}(q)$,

(5) $p(x, \xi) \neq p(x, \eta)$ for all $(x, \eta) \in \operatorname{dom}(q)$ and $(x, \xi) \in \operatorname{dom}(p) \backslash \operatorname{dom}(q)$.

Definition 11. Let $\mathcal{T}_{A, B^{*}}$ be a given pre-gap and let $X=X_{\mathcal{T}_{A, B^{*}}}$. We define the partial order $\mathcal{P}^{*}=\mathcal{P}^{*}\left(\mathcal{T}_{A, B^{*}}\right)$ as follows: Let $\mathcal{P}^{*}$ be the poset of all partial finite mappings $p: X \times \omega_{1} \rightarrow \omega$ with the following properties:

(1) $\xi<h t(x)$ for all $(x, \xi) \in \operatorname{dom}(p)$,

\footnotetext{
${ }^{3}$ Here and below $\operatorname{dom}_{0}(p)=\{x:(x, \xi) \in \operatorname{dom}(p)$ for some $\xi\}$ and $\operatorname{dom}_{1}(p)=\{\xi:(x, \xi) \in$ $\operatorname{dom}(p)$ for some $x\}$.
} 
(2) $\forall x, y \in \operatorname{dom}_{0}(p)$ and $\forall \xi<h t(x), h t(y)[(x, \xi) \in d o m(p)$ if and only if $(y, \xi) \in$ $\operatorname{dom}(p)]$,

(3) For all $x, y \in \operatorname{dom}_{0}(p)$,

(a) If $\chi(x)=\chi(y)=(\eta, 0)$ for some $\eta$, then

$$
[(x, \xi),(y, \xi) \in \operatorname{dom}(p), \xi<\Delta(x, y) \text { implies } p(x, \xi)=p(y, \xi)] .
$$

(b) If $\chi(x)=\chi(y)=(\eta, 1)$ for some $\eta$, then there exist

$$
\xi<\Delta(x, y) \text { such that } p(x, \xi) \neq p(y, \xi) \text {. }
$$

We let $p$ extend $q$ if $p$ extend $q$ as a function and

(4) $p(x, \xi)=p(y, \xi)$ for all $x, y \in \operatorname{dom}_{0}(q)$ and $\xi<\min \{h t(x), h t(y)\}, \xi \notin$ $\operatorname{dom}_{1}(q)$,

(5) $p(x, \xi) \neq p(x, \eta)$ for all $(x, \eta) \in \operatorname{dom}(q)$ and $(x, \xi) \in \operatorname{dom}(p) \backslash \operatorname{dom}(q)$.

Note that the only difference between the partial orders $\mathcal{P}$ and $\mathcal{P}^{*}$ is in condition 3 (b) of its definition. The next result contains all the information we will need to know about the partial orders $\mathcal{P}\left(\mathcal{T}_{A, B^{*}}\right)$ and $\mathcal{P}^{*}\left(\mathcal{T}_{A, B^{*}}\right)$.

Lemma 12. (A) The partial order $\mathcal{P}\left(\mathcal{T}_{A, B^{*}}\right)$ satisfies the countable chain condition for any pre-gap $\mathcal{T}_{A, B^{*}}$.

(B) The partial order $\mathcal{P}^{*}\left(\mathcal{T}_{A, B^{*}}\right)$ satisfies the countable chain condition for any pre-gap $\mathcal{T}_{A, B^{*}}$ for which $A \neq \varnothing$.

Proof. Let $\left\langle p_{\gamma}: \gamma \in \omega_{1}\right\rangle$ be given uncountable sequence of conditions in either of the forcing notions such that if $A \neq \emptyset$ then for every $\gamma \in A$ there is $x \in \operatorname{dom}_{0}\left(p_{\gamma}\right)$ such that $h t(x) \geq \gamma$ and $\pi_{1}(\chi(x))=0$. Since both proofs are similar we shall prove them at the same time and we will indicate what changes need to be done whenever is necessary. By the $\Delta$-system lemma and a counting argument, we may assume that $\left\langle\operatorname{dom}\left(p_{\gamma}\right): \gamma \in \omega_{1}\right\rangle$ form a $\Delta$-system with root $D$ and moreover $p_{\gamma} \uparrow D=p_{\gamma^{\prime}} \uparrow D$ for all $\gamma, \gamma^{\prime} \in \omega_{1}$. Let $E_{\gamma}=\chi\left[\operatorname{dom}_{0}\left(p_{\gamma}\right)\right]$.

By applying $\Delta$-system lemma to the sequence $\left\langle E_{\gamma}: \gamma \in \omega_{1}\right\rangle$ we get an uncountable set $\Xi \subseteq \omega_{1}$ and a finite set $E$ such that $\left\langle E_{\gamma}: \gamma \in \Xi\right\rangle$ forms a $\Delta$-system with root $E$. We consider two possible assumptions about this $\Delta$-system and show that in both cases we can find two compatible conditions in the $\Delta$-system.

Assumption 1: $E_{\gamma}=E$ for all $\gamma \in \Xi$.

For $\delta<\omega_{1}$, let

$$
a_{\delta}=\left\{x \uparrow \delta: x \in \operatorname{dom}_{0}\left(p_{\delta}\right), h t(x) \geqslant \delta\right\}^{4}
$$

and

$$
b_{\delta}=\left\{x \in \operatorname{dom}_{0}\left(p_{\delta}\right): h t(x)<\delta\right\} .
$$

Moreover, for $\delta<\alpha$, we let $h(\delta)$ be the minimal ordinal that dominates every ordinal of the set

$$
\left\{\Delta(x, y): x, y \in a_{\delta}, \chi(x)=\chi(y)\right\} \cup\left(\operatorname{dom}_{1}\left(p_{\delta}\right) \cap \delta\right) \cup\left\{h t(x): x \in b_{\delta}\right\} .
$$

\footnotetext{
${ }^{4}$ Here and below $x \uparrow \delta$ denote the unique predecessor of $x$ in the $\delta$-th level. We shall extend this notation to the nodes $x$ of height $<\delta$ as well by simply letting $x \uparrow \delta=x$. This way for our set $X=X_{\mathcal{T}_{A, B^{*}}}$ (and for that matter for any other set of nodes) and any $\delta<\omega_{1}$ we can define $X \uparrow \delta=\{x \uparrow \delta: x \in X\}$.
} 
By the pressing down lemma there is a stationary set $\Gamma$ of countable limit ordinals on which the mapping $h$, the mapping

$$
\delta \rightarrow \operatorname{dom}\left(p_{\delta}\right) \cap(X \uparrow \delta) \times \delta,
$$

as well as the mapping $\delta \mapsto b_{\delta}$ are constant. Let $\bar{\xi}, F$ and $b$ be the constant values, respectively. Shrinking $\Gamma$, we may assume that all $a_{\delta}(\delta \in \Gamma)$ are of some fixed size $n$. Moreover, we may assume that the function $\delta \mapsto a_{\delta}\lceil\bar{\xi}$ has a constant value $a$, and that the conditions $p_{\delta}(\delta \in \Gamma)$ generate isomorphic structures over $\bar{\xi}, F$ and $a$. Thus, we want in particular the isomorphism between the $p_{\delta}(\delta \in \Gamma)$ to respect a fixed enumeration $a_{\delta}(i)(i<n)$ of $a_{\delta}$. Moreover, the isomorphism between the conditions $p_{\gamma}$ and $p_{\delta}$ is the identity on $\bar{\xi}$ and on $a_{\delta}(i)\lceil\bar{\xi}(i<n)$, and maps nodes extending $a_{\gamma}(i)$ into nodes extending $a_{\delta}(i)$ for all $i<n$. Using Lemma 3 and Lemma 2(b) we find an uncountable subset $\Sigma \subset \Gamma$ such that for $\bar{\xi}<\gamma<\delta$ in $\Sigma$ :

(6) $a_{\gamma}(i) \uparrow \bar{\xi}=a_{\delta}(i) \uparrow \bar{\xi}$ for all $i<n$,

(7) every node of $\operatorname{dom}_{0}\left(p_{\gamma}\right)$ is either of height less or equal to $\bar{\xi}$ or it extends some $a_{\gamma}(i)(i<n)$

(8) every node of $d_{0} m_{0}\left(p_{\delta}\right)$ is either of height less or equal to $\bar{\xi}$ or it extends some $a_{\delta}(i)(i<n)$

(9) $\operatorname{dom}_{1}\left(p_{\gamma}\right) \subset \bar{\xi} \cup(\gamma, \delta)$ and $\operatorname{dom}_{1}\left(p_{\delta}\right) \subset \bar{\xi} \cup\left(\delta, \omega_{1}\right)$,

(10) $a_{\gamma}(i)$ and $a_{\delta}(j)$ are incomparable for all $i, j<n$, whenever $\chi\left(a_{\gamma}(i)\right)=$ $\chi\left(a_{\delta}(j)\right)$,

(11) $\left.\Delta\left(a_{\gamma}(i), a_{\delta}(i)\right)=\Delta\left(a_{\gamma}(j), a_{\delta}(j)\right)\right)$ for all $i, j<n$, whenever $\chi\left(a_{\gamma}(i)\right)=$ $\chi\left(a_{\delta}(i)\right)=\chi\left(a_{\gamma}(j)\right)=\chi\left(a_{\delta}(j)\right)$ and

(12) $\left.\Delta\left(a_{\gamma}(i), a_{\delta}(i)\right)<\Delta\left(a_{\gamma}(j), a_{\delta}(j)\right)\right)$ for all $i, j<n$, whenever $\chi\left(a_{\gamma}(i)\right)=$ $\chi\left(a_{\delta}(i)\right)<_{\text {lex }} \chi\left(a_{\gamma}(j)\right)=\chi\left(a_{\delta}(j)\right)$.

More specifically, this is done by a series of refinements. For example, one of these refinements is done by using Lemma 3 to get the conclusion (10). Another refinement is done by using Lemma 2(b) and the initial assumption that $T_{\xi}^{k} \prec T_{\eta}^{l}$ for $(\xi, k)=$ $\chi\left(a_{\delta}(i)\right)<_{\text {lex }} \chi\left(a_{\gamma}(j)\right)=(\eta, l)$ to get the conclusion of $(12)$.

We claim that if $\bar{\xi}<\gamma<\delta$ are in $\Sigma$, then $p_{\gamma}$ and $p_{\delta}$ are compatible. Our description of the extension of such $p_{\gamma}$ and $p_{\delta}$ depends, however, on whether $A \neq \varnothing$ or $A=\varnothing$. Let us first consider the case $A \neq \varnothing$ which applies to both parts of the Lemma. Let

$$
m=\max \left[\left(\operatorname{range}\left(p_{\gamma}\right) \cup\left(\operatorname{range}\left(p_{\delta}\right)\right] .\right.\right.
$$

Let $\left(\eta_{0}, 0\right)$ be the $<_{\text {lex }}$-maximum of the set $\left\{\chi\left(a_{\delta}(i)\right): i<n, \pi_{1}\left(\chi\left(a_{\delta}\right)\right)=0\right\}^{5}$ and let $\xi_{0}=\Delta\left(a_{\gamma}\left(i_{0}\right), a_{\delta}\left(i_{0}\right)\right)$ for some (every) $i_{0}$ such that $\chi\left(a_{\delta}\left(i_{0}\right)\right)=\left(\eta_{0}, 0\right)$.

We define a condition $p$ belonging to both versions of our pre-gap interpolation poset, by letting its domain be

$$
\operatorname{dom}\left(p_{\gamma}\right) \cup \operatorname{dom}\left(p_{\delta}\right) \cup\left\{\left(x, \xi_{0}\right): x \in \operatorname{dom}_{0}\left(p_{\gamma}\right) \cup \operatorname{dom}_{0}\left(p_{\delta}\right), h t(x)>\xi_{0}\right\},
$$

and letting

$$
\begin{gathered}
p\left(x, \xi_{0}\right)=m+1 \text { for } x \in \operatorname{dom}_{0}\left(p_{\gamma}\right), h t(x)>\xi_{0}, \text { and } \\
p\left(x, \xi_{0}\right)=m+2 \text { for } x \in \operatorname{dom}_{0}\left(p_{\delta}\right), h t(x)>\xi_{0}, \text { and } \\
p \uparrow\left(\operatorname{dom}\left(p_{\gamma}\right) \cup \operatorname{dom}\left(p_{\delta}\right)\right)=p_{\gamma} \cup p_{\delta} .
\end{gathered}
$$

\footnotetext{
${ }^{5}$ Here, $\pi_{1}:(A \cup B) \times 2 \rightarrow 2$ is the projection map $\pi_{1}(\xi, i)=i$.
} 
Note that $p$ as a function extends $p_{\gamma}, p_{\delta}$ and it clearly satisfies the conditions (1) and (2). Note moreover that

$$
\begin{gathered}
\operatorname{dom}_{1}(p)=\operatorname{dom}_{1}\left(p_{\gamma}\right) \cup \operatorname{dom}_{1}\left(p_{\delta}\right) \cup\left\{\xi_{0}\right\} \text { and that } \\
\operatorname{dom}_{0}(p)=\operatorname{dom}_{0}\left(p_{\gamma}\right) \cup \operatorname{dom}_{0}\left(p_{\delta}\right) .
\end{gathered}
$$

Let us show that $p$ satisfies clause (3) for being a condition. In order to do this, we proceed by cases:

Case 1: Consider $x \in \operatorname{dom}_{0}\left(p_{\gamma}\right)$ and $y \in \operatorname{dom}_{0}\left(p_{\delta}\right)$ with $\chi(x)=\chi(y)=(\eta, 0)$ for some $\eta$. Let $\xi<\Delta(x, y)$ be given such that $(x, \xi),(y, \xi) \in \operatorname{dom}(p)$.

Subcase $a$ : $x\left\lceil\gamma=a_{\gamma}(i)\right.$ and $y\left\lceil\delta=a_{\delta}(i)\right.$ for some $i<n$.

Then since $(\eta, 0) \leqslant_{l e x}\left(\eta_{0}, 0\right)$ using $(11)$ and $(12)$ and the definition of $\xi_{0}$ we get that

$$
\xi<\Delta(x, y)=\Delta\left(a_{\gamma}(i), a_{\delta}(i)\right) \leqslant \xi_{0} .
$$

It follows that $\xi \in\left(\operatorname{dom}_{1}\left(p_{\gamma}\right) \cap \operatorname{dom}_{1}\left(p_{\delta}\right)\right) \subset \bar{\xi}$. Let $y^{\prime}$ in $\operatorname{dom}_{0}\left(p_{\gamma}\right)$ be the copy of $y$ relative to the isomorphism between $p_{\gamma}$ and $p_{\delta}$. Then $y^{\prime}$ extends $a_{\gamma}(i)$ and $p_{\delta}(y, \xi)=$ $p_{\gamma}\left(y^{\prime}, \xi\right)$. Since $x$ also extends $a_{\gamma}(i)$ we have $\xi<\gamma \leqslant \Delta\left(x, y^{\prime}\right)$ and $\chi\left(y^{\prime}\right)=(\eta, 0)=$ $\chi(x)$. So by $3\left(\right.$ a) for the condition $p_{\gamma}$ we conclude that $p_{\gamma}(x, \xi)=p_{\gamma}\left(y^{\prime}, \xi\right)=p_{\delta}(y, \xi)$, as required.

Subcase $b$ : $x\left\lceil\gamma=a_{\gamma}(i)\right.$ and $y\left\lceil\gamma=a_{\delta}(j)\right.$ with $i \neq j<n$.

Let $y^{\prime}$ be the copy of $y$ in $\operatorname{dom}_{0}\left(p_{\gamma}\right)$ relative to the isomorphism between the conditions $p_{\gamma}$ and $p_{\delta}$. Since $y^{\prime}$ extends $a_{\gamma}(j)$ we get that $\Delta(x, y)=\Delta\left(a_{\gamma}(i), a_{\gamma}(j)\right)=\Delta\left(x, y^{\prime}\right)$, so in particular, $\xi<\bar{\xi}$. By 3(a) of $p_{\gamma}$ we infer $p_{\gamma}(x, \xi)=p_{\gamma}\left(y^{\prime}, \xi\right)=p_{\delta}(y, \xi)$.

Subcase $c: x \in b$ or $y \in b$, where $b$ is the constant value of the mapping $\delta \mapsto b_{\delta}$. Then either $x, y \in d_{d o m}\left(p_{\gamma}\right)$ or $x, y \in d o m_{0}\left(p_{\delta}\right)$. Let us focus on the case $x, y \in d o m_{0}\left(p_{\gamma}\right)$, as the other one is similar. Then this implies that $\xi<\bar{\xi}$, so in particular $(y, \xi)$ is a fixed point of the isomorphism between $p_{\gamma}$ and $p_{\gamma}$, so we get $p_{\gamma}(y, \xi)=p_{\delta}(y, \xi)$. Applying 3(a) for condition $p_{\gamma}$ we obtain $p_{\gamma}(x, \xi)=p_{\gamma}(y, \xi)$. This finishes the proof in Case 1.

Case 2: Consider the case $x \in \operatorname{dom}_{0}\left(p_{\gamma}\right), y \in \operatorname{dom}_{0}\left(p_{\delta}\right)$ and $\chi(x)=\chi(y)=(\eta, 1)$ for some $\eta$.

Subcase $a: x\left\lceil\gamma=a_{\gamma}(i), y\left\lceil\delta=a_{\delta}(i)\right.\right.$ for some $i<n$.

Since $\left(\eta_{0}, 0\right)<_{\text {lex }}(\eta, 1)$, it follows from $(12)$ and the definition of $\xi_{0}$ that $\Delta(x, y)=$ $\Delta\left(a_{\gamma}(i), a_{\delta}(i)\right)>\xi_{0}$. Moreover $p\left(x, \xi_{0}\right)=m+1 \neq m+2=p\left(y, \xi_{0}\right)$ so $\xi_{0}$ is a witness for clause 3(b) (for both partial orders).

Subcase $b: x\left\lceil\gamma=a_{\gamma}(i), y\left\lceil\delta=a_{\delta}(j)\right.\right.$ with $i \neq j<n$.

Then $\Delta(x, y)=\Delta\left(a_{\gamma}(i), a_{\gamma}(j)\right)=\Delta\left(a_{\delta}(i), a_{\delta}(j)\right)$ which is less than $\bar{\xi}$. Let $x^{\prime}$ and $y^{\prime}$ be the copies of $x$ and $y$ relative to the isomorphism between $p_{\gamma}$ and $p_{\delta}$, respectively. Note that $x^{\prime}$ extends $a_{\delta}(i)$ and $y^{\prime}$ extends $a_{\gamma}(j)$. Since $p_{\gamma}$ is a condition there exists $\eta<\Delta\left(x, x^{\prime}\right)=\Delta\left(y, y^{\prime}\right)$ such that $p_{\gamma}(x, \eta) \neq p_{\gamma}\left(x^{\prime}, \eta\right)$. Since the isomorphism between $p_{\gamma}$ and $p_{\delta}$ is the identity for elements less than $\bar{\xi}$ we get that $\eta \in d o m_{1}\left(p_{\delta}\right)$. Moreover, $p_{\gamma}(x, \eta)=p_{\delta}\left(x^{\prime}, \eta\right)$ and $p_{\gamma}\left(y^{\prime}, \eta\right)=p_{\delta}(y, \eta)$ which implies that $p_{\gamma}(x, \eta) \neq p_{\delta}(y, \eta)$ so $\eta$ is a witness for $3(\mathrm{~b})$.

Subcase $c: x \in b$ or $y \in b$, where $b$ is the constant value of the mapping $\delta \mapsto b_{\delta}$. Then either $x, y \in \operatorname{dom}_{0}\left(p_{\gamma}\right)$ or $x, y \in \operatorname{dom}_{0}\left(p_{\delta}\right)$. Since both cases are similar let us consider only the case $x, y \in \operatorname{dom}_{0}\left(p_{\gamma}\right)$. Let $y^{\prime}$ be the copy of $y$ relative to the isomorphism 
between $p_{\gamma}$ and $p_{\delta}$. By $3(\mathrm{~b})$ for the condition $p_{\gamma}$ we can find $\eta<\Delta(x, y)$ so that $p_{\gamma}(x, \eta) \neq p_{\gamma}\left(y^{\prime}, \eta\right)=p_{\delta}(y, \eta)$, finishing the proof in Case 2 .

In case $A=\varnothing$ the construction of the extension of $p_{\gamma}$ and $p_{\delta}$ is done in a similar manner so we just indicate the change that is needed. To this end, let $\left(\eta_{0}, 1\right)$ be the $<_{l e x}$-minimum of the set $\left\{\chi\left(a_{\delta}(i)\right): i<n, \pi_{1}\left(\chi\left(a_{\delta}\right)\right)=1\right\}$ and let $\xi_{0}=\Delta\left(a_{\gamma}\left(i_{0}\right), a_{\delta}\left(i_{0}\right)\right)$ for some (every) $i_{0}$ such that $\chi\left(a_{\delta}\left(i_{0}\right)\right)=\left(\eta_{0}, 1\right)$. The rest of the construction follows that of the case $A \neq \varnothing$ and is left to the reader.

We are left with proving that $p$ extends both $p_{\gamma}$ and $p_{\delta}$ and we again give the full details only in the case of the construction coming from the case $A \neq \emptyset$ leaving the other analogous case to the interested reader. Given $x, y \in d o m_{0}\left(p_{\gamma}\right)$ or $x, y \in$ $\operatorname{dom}_{0}\left(p_{\delta}\right)$ we have that $p\left(x, \xi_{0}\right)=m+1=p\left(y, \xi_{0}\right)$ or $p\left(x, \xi_{0}\right)=m+2=p\left(y, \xi_{0}\right)$, respectively, where $\xi_{0}$ is the only new member of $\operatorname{dom}_{1}(p) \backslash \operatorname{dom}_{1}\left(p_{\gamma}\right)$ and the only element of $d_{0} m_{1}\left(p_{\delta}\right)$. On the other hand, given $x \in d o m_{0}\left(p_{\gamma}\right)$ or $x \in d o m_{0}\left(p_{\delta}\right)$ we have that $p\left(x, \xi_{0}\right)=m+1>p(x, \eta)$ for all $(x, \eta) \in \operatorname{dom}\left(p_{\gamma}\right)$ or $p\left(x, \xi_{0}\right)=m+2>p(x, \eta)$ for all $(x, \eta) \in \operatorname{dom}\left(p_{\gamma}\right)$ and all $(x, \eta) \in \operatorname{dom}\left(p_{\delta}\right)$. Thus, $p$ satisfies clauses (4) and (5). This finishes the proof of that under the Assumption 1 the given sequence $\left\langle p_{\gamma}: \gamma \in \omega_{1}\right\rangle$ has two compatible members in both versions of the poset .

Assumption 2: $E_{\gamma} \backslash E \neq \varnothing$ for all $\gamma \in \Xi$.

Let us consider the reduction $\left\langle p_{\gamma}^{E}: \gamma \in \Xi\right\rangle$ of the sequence $\left\langle p_{\gamma}: \gamma \in \Xi\right\rangle$ to the suborder $\mathcal{P}\left(\mathcal{T}_{A \cap E,(B \cap E)^{*}}\right)\left(\right.$ or $\left.\mathcal{P}^{*}\left(\mathcal{T}_{A \cap E,(B \cap E)^{*}}\right)\right)$, where

$$
p_{\gamma}^{E}=p_{\gamma} \uparrow\left\{(x, \xi) \in \operatorname{dom}\left(p_{\gamma}\right): \chi(x) \in E\right\} .
$$

By the work done under the Assumption 1, we know that the posets $\mathcal{P}\left(\mathcal{T}_{A \cap E,(B \cap E)^{*}}\right)$ and $\mathcal{P}^{*}\left(\mathcal{T}_{A \cap E,(B \cap E)^{*}}\right)$ satisfy the countable chain condition, so there exist $\gamma, \delta \in \Xi$ and $r \in \mathcal{P}\left(\mathcal{T}_{A \cap E,(B \cap E)^{*}}\right)\left(\right.$ or $\left.\mathcal{P}^{*}\left(\mathcal{T}_{A \cap E,(B \cap E)^{*}}\right)\right)$ such that $r \leqslant p_{\gamma}^{E}, p_{\delta}^{E}$. We can amalgamate $r$ with $p_{\gamma}, p_{\delta}$ to a new condition $q$ of $\mathcal{P}\left(\mathcal{T}_{A \cap E,(B \cap E)^{*}}\right)\left(\right.$ or $\left.\mathcal{P}^{*}\left(\mathcal{T}_{A \cap E,(B \cap E)^{*}}\right)\right)$ as follows. First of all note that $r \cup p_{\gamma} \cup p_{\delta}$ is a function. Let

$$
n=\max \left[\operatorname{rang}(r) \cup \operatorname{rang}\left(p_{\gamma}\right) \cup \operatorname{rang}\left(p_{\delta}\right] .\right.
$$

Let $q$ be the function with domain

$$
\left\{(x, \xi): x \in \operatorname{dom}_{0}\left(r \cup p_{\gamma} \cup p_{\delta}\right), \xi \in \operatorname{dom}_{1}\left(r \cup p_{\gamma} \cup p_{\delta}\right) \xi<h t(x)\right\} .
$$

Defined by $q\left\lceil\operatorname{dom}(r)=r, q\left\lceil\operatorname{dom}\left(p_{\gamma}\right)=p_{\gamma}, q\left\lceil\operatorname{dom}\left(p_{\delta}\right)=p_{\delta}\right.\right.\right.$ and $q(x, \xi)=$ $n+1$ for all $(x, \xi) \in \operatorname{dom}(q) \backslash\left(\operatorname{dom}(r) \cup \operatorname{dom}\left(p_{\gamma}\right) \cup \operatorname{dom}\left(p_{\delta}\right)\right.$. Since the clause (3) is only meaningful for pairs $x, y \in \operatorname{dom}_{0}(q)$ so that $\chi(x)=\chi(y)$, it follows from our construction that it only applies to pairs $x, y$ so that either $x, y \in \operatorname{dom}_{0}\left(p_{\gamma}\right)$ or $x, y \in \operatorname{dom}_{0}\left(p_{\delta}\right)$ or $\chi(x)=\chi(y) \in A$. In the later case clause (3) holds since $x, y \in \operatorname{dom}_{0}(r)$ and $r$ is a condition of $\mathcal{P}\left(\mathcal{T}_{A \cap E,(B \cap E)^{*}}\right)\left(\right.$ or $\left.\mathcal{P}^{*}\left(\mathcal{T}_{A \cap E,(B \cap E)^{*}}\right)\right)$. Thus, $q$ is a condition. Let us now show that $q$ extend both $p_{\gamma}$ and $p_{\delta}$. Consider $x, y \in \operatorname{dom}_{\left(p_{\gamma}\right)}$ or $x, y \in \operatorname{dom}_{0}\left(p_{\delta}\right)$ it follows from the definition of $q$ that $q(x, \xi)=n+1=q(y, \xi)$ for all $\xi \notin d o m_{1}\left(p_{\gamma}\right)$ or $\xi \notin d o m_{1}\left(p_{\delta}\right)$. Moreover, $q(x, \xi)=n+1>q(x, \eta)$ for all $\xi \notin \operatorname{dom}_{1}\left(p_{\gamma}\right)$ and $\eta \in \operatorname{dom}_{1}\left(p_{\gamma}\right)$, or $\xi \notin \operatorname{dom}_{1}\left(p_{\delta}\right)$ and $\eta \in \operatorname{dom}_{1}\left(p_{\delta}\right)$. This finishes the proof. 


\section{Pre-Gaps in $\langle\mathcal{C}, \preceq\rangle$ under $M A_{\omega_{1}}$}

In this section we show that sufficiently generic filters of the posets $\mathcal{P}\left(\mathcal{T}_{A, B^{*}}\right)$ and $\mathcal{P}^{*}\left(\mathcal{T}_{A, B^{*}}\right)$ will give us coherent A-trees that interpolate the pre-gap $\mathcal{T}_{A, B^{*}}=\left\langle T_{\xi}^{0}, T_{\eta}^{1}\right.$ : $\xi \in A, \eta \in B\rangle$.

Lemma 13. Let $\mathcal{T}_{A, B^{*}}$ be a pre-gap of $\langle\mathcal{C}, \prec\rangle$ indexed by $A, B \subseteq \omega_{1}$ and let $\mathcal{P}=$ $\mathcal{P}\left(\mathcal{T}_{A, B^{*}}\right)$ or $\mathcal{P}=\mathcal{P}^{*}\left(\mathcal{T}_{A, B^{*}}\right)$. Then for all $x_{0} \in X$, the set

$$
\mathcal{D}_{x_{0}}=\left\{p \in \mathcal{P}: x_{0} \in \operatorname{dom}_{0}(p)\right\}
$$

is dense open in $\mathcal{P}$.

Proof. Since the proof for $\mathcal{P}=\mathcal{P}^{*}\left(\mathcal{T}_{A, B^{*}}\right)$ is quite similar we focus on the case $\mathcal{P}=$ $\mathcal{P}\left(\mathcal{T}_{A, B^{*}}\right)$. Fix $p \in \mathcal{P}$. If $x_{0} \in \operatorname{dom}_{0}(p)$ we are done, so let us assume $x_{0} \notin d o m_{0}(p)$. We proceed by considering two cases and some subcases.

Case 1: If $\chi\left(x_{0}\right)=(\xi, 1)$ for some $\xi$. Let $n=\max (\operatorname{rang}(p))$.

Subcase a: If there is a $x \in \operatorname{dom}_{0}(p)$ such that $\chi(x)=\chi\left(x_{0}\right)$. Set

$$
\bar{\xi}=\min \left\{\Delta\left(x_{0}, x\right): x \in\left(\operatorname{dom}_{0}(p) \backslash\left\{x_{0}\right\}\right), \chi\left(x_{0}\right)=\chi(x)\right\} .
$$

By (iv) $\bar{\xi}>\omega$ so we can find $\xi_{0}<\bar{\xi}, \xi_{0} \notin \operatorname{dom}_{1}(p)$. Let $q$ be a map with domain equal to

$$
\begin{gathered}
\operatorname{dom}(p) \cup\left\{\left(x, \xi_{0}\right): x \in \operatorname{dom}_{0}(p), \xi_{0}<h t(x)\right\} \\
\cup\left\{\left(x_{0}, \xi_{0}\right)\right\} \cup\left\{\left(x_{0}, \xi\right): \xi \in \operatorname{dom}_{1}(p), \xi<h t\left(x_{0}\right)\right\} .
\end{gathered}
$$

Define $q$ by $q\lceil\operatorname{dom}(p)=p$,

$$
\begin{gathered}
q\left(x, \xi_{0}\right)=n+2 \text { for } x \in \operatorname{dom}_{0}(p), \text { and } \\
q\left(x_{0}, \xi\right)=q\left(x_{0}, \xi_{0}\right)=n+1 \text { for all } \xi \in \operatorname{dom}_{1}(p), \xi<h t\left(x_{0}\right) .
\end{gathered}
$$

Let us show that $q$ is a condition in the partial order $\mathcal{P}$. It should be clear from our definition of $q$ that clauses (1) and (2) holds. Consider $x, y \in \operatorname{dom}_{0}(q)$ with $\chi(x)=\chi(y)$ and $\pi_{1}(\chi(x))=0^{6}$. Let $\xi<\Delta(x, y), \xi \in d o m_{1}(q)$ be given. Then either $\xi \in \operatorname{dom}_{1}(p)$ which implies $q(x, \xi)=q(y, \xi)$ since $(x, \xi),(y, \xi) \in \operatorname{dom}(p)$, or $\xi=\xi_{0}$ which implies $q\left(x, \xi_{0}\right)=n+2=q\left(y, \xi_{0}\right)$. This shows that clause 3(a) holds. To verify clause 3(b), let $x, y \in \operatorname{dom}_{0}(q)$ be given so that $\chi(x)=\chi(y)$ and $\pi_{1}(\chi(x))=1$. If $x, y \in \operatorname{dom}_{0}(p)$ then using the fact that $p$ is a condition, we can find $\eta \leq \Delta(x, y)$ in $\operatorname{dom}_{1}(p)$ so that $p(x, \eta) \neq p(y, \eta)$. On the other hand, if $x_{0}=x$ then $q\left(x_{0}, \xi_{0}\right)=$ $n+1 \neq n+2=q\left(y, \xi_{0}\right)$. It follows that $q$ is a condition which belongs to $\mathcal{D}_{x_{0}}$. Let us verify that $q$ extends $p$. First of all, by its very definition, $q$ extends $p$ as a function. Note that for $x, y \in \operatorname{dom}_{0}(p)$ we have $q\left(x, \xi_{0}\right)=n+2=q\left(y, \xi_{0}\right)$ with $\xi_{0}$ as the only element in $\operatorname{dom}_{1}(q) \backslash \operatorname{dom}_{1}(p)$. Moreover, $q\left(x, \xi_{0}\right)=n+2>q(x, \xi)$ for all $(x, \xi) \in \operatorname{dom}(p)$. So this checks that $q$ is a condition extending $p$.

Subcase b: There is no $x \in \operatorname{dom}_{0}(p)$ so that $\chi\left(x_{0}\right)=\chi(x)$. Then let $q$ be the function with domain

$$
\operatorname{dom}(p) \cup\left\{\left(x_{0}, \xi\right): \xi \in \operatorname{dom}_{1}(p), \xi<h t\left(x_{0}\right)\right\}
$$

defined by $q\lceil\operatorname{dom}(p)=p$ and

$$
q\left(x_{0}, \xi\right)=n+1 \text { for all } \xi \in \operatorname{dom}_{1}(p), \xi<h t\left(x_{0}\right) .
$$

\footnotetext{
${ }^{6}$ Recall that $\pi_{1}:(A \cup B) \times 2 \rightarrow 2$ is the projection map $\pi_{1}(\xi, i)=i$.
} 
It should be clear that $q$ is a condition belonging to $\mathcal{D}_{x_{0}}$ and extending $p$.

Case 2: $\chi\left(x_{0}\right)=(\xi, 0)$ for some $\xi$. Let $n=\max (\operatorname{rang}(p))$.

Subcase $a$ : There is $x \in \operatorname{dom}_{0}(p)$ such that $\chi\left(x_{0}\right)=\chi(x)$. Pick $x_{0}^{\prime} \in \operatorname{dom}_{0}(p)$ such that $\chi\left(x_{0}^{\prime}\right)=\chi\left(x_{0}\right)$ and

$$
\Delta\left(x_{0}, x_{0}^{\prime}\right)=\max \left\{\Delta\left(x_{0}, x\right): x \in \operatorname{dom}_{0}(p), \chi\left(x_{0}\right)=\chi(x)\right\} .
$$

Let $q$ be map with domain

$$
\operatorname{dom}(p) \cup\left\{\left(x_{0}, \xi\right): \xi \in \operatorname{dom}_{1}(p), \xi<h t\left(x_{0}\right)\right\} .
$$

Define $q$ by $q\lceil\operatorname{dom}(p)=p$ and

$$
\begin{gathered}
q\left(x_{0}, \xi\right)=p\left(x_{0}^{\prime}, \xi\right) \text { for } \xi \in \operatorname{dom}_{1}(p) \cap \Delta\left(x_{0}, x_{0}^{\prime}\right) \text { and } \\
q\left(x_{0}, \xi\right)=n+1 \text { for } \xi \in \operatorname{dom}_{1}(p) \cap\left[\Delta\left(x_{0}, x_{0}^{\prime}\right), h t\left(x_{0}\right)\right) .
\end{gathered}
$$

Let us show that $q$ is a condition. First note that $q$ satisfies clauses (1), (2) and 3 (b) by definition. In order to verify clause $3(\mathrm{a})$ let $x, y \in \operatorname{dom}_{0}(q)$ be given so that $\chi(x)=\chi(y)$ and $\pi_{1}(\chi(x))=0$. Let $\xi<\Delta(x, y), \xi \in \operatorname{dom}_{1}(q)$ be given. Then either $x, y \in \operatorname{dom}_{0}(p)$ and therefore $q(x, \xi)=q(y, \xi)$ since $(x, \xi),(y, \xi) \in p$ and $p$ is a condition, or $x=x_{0}$ which implies $\xi<\Delta\left(x_{0}, y\right)=\Delta\left(x_{0}^{\prime}, y\right)$ and therefore $q(y, \xi)=q\left(x_{0}^{\prime}, \xi\right)=q\left(x_{0}, \xi\right)$. This checks that $q$ is indeed a condition. Note also that $q$ extends $p$ as clauses (5) and (6) are vacuously true. Therefore $q$ is a condition belonging to $\mathcal{D}_{x_{0}}$ and extending $p$.

Subcase b: There is no $x \in \operatorname{dom}_{0}(p)$ so that $\chi\left(x_{0}\right)=\chi(x)$. Then let $q$ be the condition with domain

$$
\operatorname{dom}(p) \cup\left\{\left(x_{0}, \xi\right): \xi \in \operatorname{dom}_{1}(p), \xi<h t\left(x_{0}\right)\right\}
$$

be defined by $q \uparrow \operatorname{dom}(p)=p$ and

$$
q\left(x_{0}, \xi\right)=n+1 \text { for all } \xi \in \operatorname{dom}_{1}(p) \text { such that } \xi<h t\left(x_{0}\right) .
$$

Then it is readily seen that $q$ is a condition which belongs to $\mathcal{D}_{x_{0}}$ and extends $p$.

Lemma 14. Let $\mathcal{T}_{A, B^{*}}$ be a pre-gap of $\langle\mathcal{C}, \prec\rangle$ indexed by $A, B \subseteq \omega_{1}$ and let $\mathcal{P}=$ $\mathcal{P}\left(\mathcal{T}_{A, B^{*}}\right)$ or $\mathcal{P}=\mathcal{P}^{*}\left(\mathcal{T}_{A, B^{*}}\right)$. Then for all $\left(x_{0}, \xi_{0}\right) \in X \times \omega_{1}$ with $\xi_{0}<\operatorname{ht}\left(x_{0}\right)$, the set

$$
\mathcal{D}_{\left(x_{0}, \xi_{0}\right)}=\left\{p \in \mathcal{P}:\left(x_{0}, \xi_{0}\right) \in \operatorname{dom}(p)\right\}
$$

is dense and open in $\mathcal{P}$.

Proof. Let $p \in \mathcal{P}$ be given. By Lemma 13, we can find a condition $p^{\prime}$ extending $p$ so that $x_{0} \in \operatorname{dom}_{0}\left(p^{\prime}\right)$. Let $n=\max \left(\operatorname{rang}\left(p^{\prime}\right)\right)$. Set $q$ to be the function with domain

$$
\operatorname{dom}\left(p^{\prime}\right) \cup\left\{\left(x, \xi_{0}\right): x \in \operatorname{dom}_{0}\left(p^{\prime}\right), \xi_{0}<h t(x)\right\}
$$

be defined by $q \uparrow \operatorname{dom}\left(p^{\prime}\right)=p^{\prime}$ and

$$
q\left(x, \xi_{0}\right)=n+1 \text { for all } x \in \operatorname{dom}_{0}\left(p^{\prime}\right) \text { such that } \xi_{0}<h t(x) .
$$

Let us verify that $q$ is a condition. It should be clear that clauses (1) and (2) holds. Let $x, y \in \operatorname{dom}_{0}(q)$ be given so that $\chi(x)=\chi(y)$ and $\pi_{1}(\chi(x))=1$. Since $x, y \in$ $\operatorname{dom}_{0}\left(p^{\prime}\right)$ and $p$ is a condition, we can find $\eta \in \operatorname{dom}_{1}\left(p^{\prime}\right)$ such that $\eta<\Delta(x, y)$ and $p(x, \eta) \neq p(y, \eta)$. On the other hand, given $x, y \in \operatorname{dom}_{0}(q), \xi \in \operatorname{dom}_{1}(q)$ such that $\chi(x)=\chi(y), \pi_{1}(\chi(x))=0$ and $\xi<\Delta(x, y)$ then either $\xi \in d o m_{1}\left(p^{\prime}\right)$ which would imply $q(x, \xi)=q(y, \xi)$ since $(x, \xi),(y, \xi) \in \operatorname{dom}\left(p^{\prime}\right)$ and $p^{\prime}$ is a condition, or $\xi=\xi_{0}$ 
in which case $q\left(x, \xi_{0}\right)=n+1=q\left(y, \xi_{0}\right)$. We are left to prove that $q$ extends $p^{\prime}$. First of all note that $q\left(x, \xi_{0}\right)=n+1=q\left(y, \xi_{0}\right)$ for all $x, y \in \operatorname{dom}_{0}(q)$. It follows that $\xi_{0}<h t(x), h t(y)$, where $\xi_{0}$ is the only element of $d o m_{1}(q) \backslash d o m_{1}\left(p^{\prime}\right)$. Moreover, $q\left(x, \xi_{0}\right)=n+1>q(x, \xi)$ for all $(x, \xi) \in \operatorname{dom}\left(p^{\prime}\right)$. This finishes the proof that $D_{\left(x_{0}, \xi_{0}\right)}$ is open and dense in $\mathcal{P}$.

We are now in position to state the main result of this section.

Theorem 15. Assume $\mathrm{MA}_{\omega_{1}}$. For every pre-gap

$$
\mathcal{T}_{A, B^{*}}=\left\langle T_{\xi}^{0}, T_{\eta}^{1}: \xi \in A, \eta \in B\right\rangle
$$

in the class $\langle\mathcal{C}, \prec\rangle$ of coherent $A$-trees indexed by some $A, B \subseteq \omega_{1}$ there is a coherent $A$-tree $T$ such that $T_{\eta}^{0} \preceq T \preceq T_{\xi}^{1}$ for all $\eta \in A, \xi \in B$. Moreover, if $A \neq \varnothing$ we can find a tree $T$ that satisfies $T_{\eta}^{0} \preceq T \prec T_{\xi}^{1}$ for $\eta \in A, \xi \in B$, or in other words, we can make the second inequality strict.

Proof. By $\mathrm{MA}_{\omega_{1}}$ we can find a filter $G$ intersecting the dense-open sets $D_{\left(x_{0}, \xi\right)}$ for all $x_{0} \in X$ and $\xi<h t\left(x_{0}\right)$. Let $F=\bigcup G$. Then for each $x \in X$ we can define the corresponding fiber map $f_{x}: h t(x) \rightarrow \omega$ by $f_{x}(\xi)=F(x, \xi)$. Let $T$ be the downward closure of the set $\left\{f_{x}: x \in X\right\}$. By clauses (4) and (5) of the definition of the forcing notion, the tree $T$ is a coherent A-tree. Now the following claim finishes the proof.

Claim 1: $T_{\xi}^{0} \preceq T \preceq T_{\eta}^{1}$ for all $\xi \in A$ and $\eta \in B$.

To see this, for each $\xi \in A$, we set

$$
X_{\xi}^{0}=\{x \in X: \chi(x)=(\xi, 0)\} .
$$

Consider the map $\varphi_{\xi}: X_{\xi}^{0} \rightarrow T$ defined by $\varphi_{\xi}(x)=f_{x}$. Then from the clause $3(\mathrm{a})$ it follows that for all $\xi \in A$, the map $\varphi_{\xi}$ is Lipschitz map on its uncountable domain $X_{\xi}^{0} \subseteq T_{\xi}^{0}$. Using Lemma 2(a), we obtain the inequalities $T_{\xi}^{0} \preceq T$ for all $\xi \in A$. For $\eta \in B$ let

$$
Y_{\eta}=\left\{f_{x}: \chi(x)=(\eta, 1)\right\} .
$$

Now define a map $\psi_{\eta}: Y_{\eta} \rightarrow T_{\eta}^{1}$ as follows: $\psi_{\eta}\left(f_{x}\right)=x$. Then by $3(\mathrm{~b})$ we infer $\psi_{\eta}$ is a Lipschitz map on its uncountable domain $Y_{\eta} \subseteq T$. Using Lemma 2(a) again, we conclude that $T \preceq T_{\eta}^{1}$ for all $\eta \in B$. Finally note that if $A$ is non-empty then from clause 3(b) for the poset $P^{*}$ and Lemma 2(b) we can infer that $T \prec T_{\eta}^{1}$ for all $\eta \in B$.

Remark 16. We can not expect to find a tree where the strict inequality in the conclusion of Theorem 14 holds for all type of pre-gaps. In particular, in view of Lemma 6 , this is not possible for the $\langle 1,1\rangle$ pre-gap $\left\langle T, T^{(1)}\right\rangle$ for any coherent Aronsajn tree $T$.

To avoid this kind of difficulties, we shall pass to the quotient structure $\langle\mathcal{C} / \mathbb{Z}, \prec\rangle$, where we let $\mathcal{C} / \mathbb{Z}$ be the collection of all classes of the equivalence relation

$$
S \equiv T(\bmod \mathbb{Z}) \text { iff there is } n \in \omega \text { so that } T \equiv S^{(n)} \text { or } T^{(n)} \equiv S
$$

induced by the shift, and where use the same notation for the ordering which $\prec$ induces on this quotient structure. We consider this quotient ordering in the next section. 


\section{Pre-Gaps in $\langle\mathcal{C}, \preceq\rangle$ under PFA}

We are now in position to state and prove the main theorem of this paper.

Theorem 17. Assume PFA. There are no $\left\langle\kappa, \lambda^{*}\right\rangle$-gaps in $\langle\mathcal{C}, \preceq\rangle$ whenever $\kappa, \lambda \in$ $\left\{\varnothing, 1, \omega, \omega_{1}\right\}$ and $\left\langle\kappa, \lambda^{*}\right\rangle \neq\langle 1,1\rangle$.

Proof. We split the proof in the following four cases.

Case 1: There are no $\langle\varnothing, 1\rangle$ and $\langle 1, \varnothing\rangle$ gaps.

This is equivalent to the non existence of minimal or maximal coherent A-trees, which is the content of Theorem 7 .

Case 2: There are no gaps of type $\left\langle\kappa, \lambda^{*}\right\rangle$-gaps for $\lambda, \kappa \in\left\{\varnothing, \omega, \omega_{1}\right\}$.

Note that if $T$ is the tree given by the conclusion of Theorem 15, then $T$ fills any pre-gap of this type.

Case 3: There are no gaps of the form $\langle\omega, 1\rangle$ nor $\left\langle\omega_{1}, 1\right\rangle$.

This is similar to Case 2 where we use Theorem 15 again and moreover we use the extra assumption that $A$ is non-empty.

Case 4: There are no $\left\langle 1, \omega^{*}\right\rangle$ nor $\left\langle 1, \omega_{1}^{*}\right\rangle$ gaps. Since the two cases are similar we focus on the $\left\langle 1, \omega_{1}^{*}\right\rangle$-pre-gaps. Consider a pre-gap

$$
S \prec \ldots \prec S_{\xi} \prec \ldots \prec S_{1} \prec S_{0}\left(\xi<\omega_{1}\right)
$$

of coherent A-trees. Note that the existence of the tree which fills the pregap is not immediate from Theorem 15, since nothing rules out the possibility that the tree $T$ given by Theorem 15 is equal to $S$. Note however that since there are no coherent A-trees between $S$ and its shift $S^{(1)}$, we have that $S^{(1)} \prec S_{\xi}$ for all $\xi<\omega_{1}$. Repeating this over all other finite shifts $S^{(n)}$ of $S$, we get that

$$
S=S^{(0)} \prec S^{(1)} \prec \ldots \prec S^{(n)} \prec \ldots \prec S_{\xi} \prec \ldots \prec S_{1} \prec S_{0}\left(n \in \omega, \xi<\eta \in \omega_{1}\right) .
$$

It follows from Case 2 that there is a tree $T$ such that

$$
S^{(n)} \prec T \prec S_{\xi} \text { for all }\left(n<\omega, \xi<\omega_{1}\right) .
$$

This $T$ fills the $\left\langle 1, \omega_{1}^{*}\right\rangle$ pre-gap.

Our first corollary of Theorem 17 answers Question 9.9 of [4].

Corollary 18. Assuming PFA, the chain $\langle\mathcal{C}, \preceq\rangle$ of coherent A-trees has the cofinality and the coinitiality equal to $\omega_{2}$.

Proof. This uses the additional fact that, under PFA, the two numbers are bounded by $\omega_{2}$ since $|\mathcal{C}|=2^{\aleph_{1}}=\aleph_{2}$.

We finish this paper with a complete description of the quotient ordering $\langle\mathcal{C} / \mathbb{Z}, \preceq\rangle$ under the assumption of PFA.

Corollary 19. Assuming PFA, the ordering $\langle\mathcal{C} / \mathbb{Z}, \preceq\rangle$ is the unique $\aleph_{2}$-saturated linear order of cardinality $\aleph_{2}$.

Proof. The result is an immediate consequence of Theorem 17 and the fact that $|\mathcal{C} / \mathbb{Z}|=|\mathcal{C}|=2^{\aleph_{1}}=\aleph_{2}$ under PFA. 


\section{References}

[1] G. Kurepa, Ensembles ordonnés et ramifies, Acad. Serbe Sci. Publ. Inst. Math. 4 (1935) 1-138.

[2] R. Laver, Better-quasi-orderings and a class of trees, in Studies in foundations and combinatorics, Vol. 1 of Adv. in Math. Suppl. Stud., 31-48, Academic Press, New York (1978).

[3] C. Martinez-Ranero, The Aronszajn lines are well-quasi-ordered by embeddability (2009). Submitted to Fund. Math.

[4] J. T. Moore, Structural analysis of Aronszajn trees, in Logic Colloquium 2005, Vol. 28 of Lect. Notes Log., 85-106, Assoc. Symbol. Logic, Urbana, IL (2008).

[5] M. Suslin, Problem 3, Fund Math. 1 (1920) 223.

[6] S. Todorcevic, Lipschitz maps on trees, J. Inst. Math. Jussieu 6 (2007), no. 3, 527-556.

[7] - Walks on ordinals and their characteristics, Vol. 263 of Progress in Mathematics, Birkhäuser Verlag, Basel (2007), ISBN 978-3-7643-8528-6.

Department of Mathematics, University of Toronto, Room 6290, 40 St. George street, Toronto, Ontario, Canada M5S 2E4

E-mail address: azarel@math.toronto.edu

Department of Mathematics, University of Toronto, Room 6290, 40 St. George street, Toronto, Ontario, Canada M5S 2E4

E-mail address: stevo@math.toronto.edu

Institute Se Mathématiques de Jussieu, CNRS-UMR 7586, 75205 Paris Cedex 13, France

E-mail address: stevo@math.jussieu.fr 